\title{
Uncovering Flickering in Time-Resolved Spectroscopy for the Nova-like Variable UX Ursa Majoris
}

\author{
James C. White II \\ Department of Physics and Astronomy, Middle Tennessee State \\ University, Murfreesboro, TN 37132 USA
}

\begin{abstract}
.
The flickering phenomenon in cataclysmic variables (CVs) is manifest photometrically as variations in system brightness with amplitudes up to about $1 \mathrm{mag}$ and on timescales from seconds to tens of minutes. Although a pronounced characteristic of CVs, flickering has been studied, in general, only tangentially and in the context of overall CV photometric properties. It is usually uncovered in CV photometry, yet my Doppler tomographic analysis of data collected on the KPNO 2.1-meter telescope in 1982 by Schlegel, Honeycutt, and Kaitchuck (1983) for the nova-like variable UX Ursa Majoris suggests that flickering also exists in this system's spectroscopy.

Using the integrated continuum flux from the UX UMa observations and Bruch's (1992) methodology for classifying high- and low-flicker data, I constructed two data subsets corresponding to high- and low-flicker states. I generated Doppler tomograms of these high- and low-flicker states and interpret differences between the tomograms as spectroscopic signatures of flickering in the system.

In UX UMa's $\mathrm{H} \beta$ Doppler tomograms, a small, faint, incomplete ring of emission is visible for both flickering states. Dominating ring emission is strong emission in the $\left(+V_{x},+V_{y}\right)$ quadrant. The usual location for $S-$ wave emission, in the $\left(-V_{x},+V_{y}\right)$ quadrant, is here the region of faintest emission. Differenced Doppler tomograms, produced by subtracting from the tomograms the symmetric ring-emission component, show clear differences between the high- and low-flicker states. Further, these early results suggest Doppler tomography may be a useful technique for studying the secondary stars in CVs imaged in this manner. Enhanced chromospheric activity of the red dwarf companion is clearly visible in the high-flickering state tomogram at $\left(0, \mathrm{~K}_{\mathrm{rd}}\right)$ and may, therefore, afford investigators the opportunity to estimate the chromospheric flux and radial velocity of the secondary star.
\end{abstract}

\section{References}

Bruch, A. $1992, A \& A, 266,237$

Schlegel, E. M., Honeycutt, R. K., \& Kaitchuck, R. H. 1983, ApJS, 53, 397 